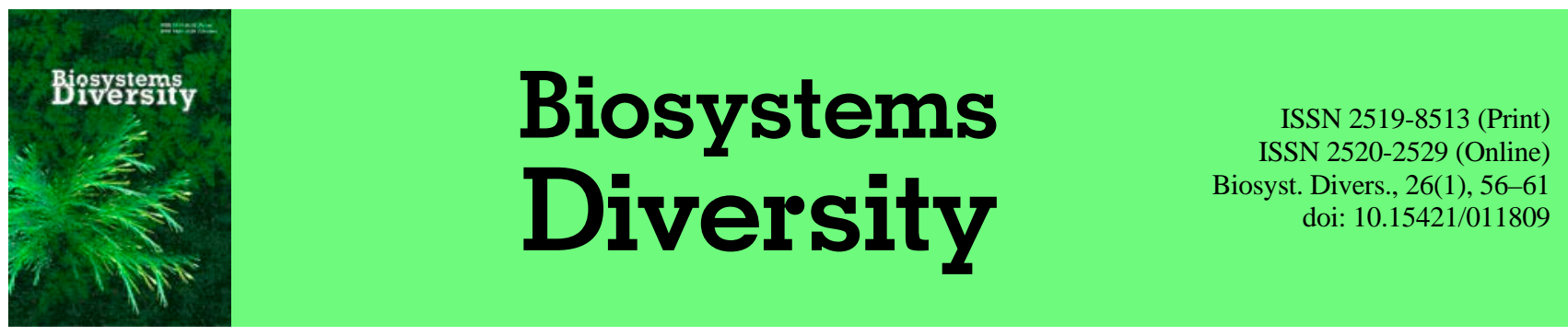

\title{
Ethno-botanical and geo-referenced profiling of medicinal plants of Nawagai Valley, District Buner (Pakistan)
}

\author{
S. U. Zaman, K. Ali, W. Khan, M. Ali, T. Jan, M. Nisar \\ University of Malakand, Chakdara, Pakistan
}

Article info

Received 09.02.2018

Received in revised form 23.02.2018

Accepted 25.02.2018

Department of Botany, University of Malakand Chakdara, Dir Lower,

Pakistan.

E-mail: kishwarali@live.com

\begin{abstract}
Zaman, S. U., Ali, K., Khan, W., Ali, M., Jan, T., \& Nisar, M. (2018). Ethno-botanical and geo-referenced profiling of medicinal plants of Nawagai Valley, District Buner (Pakistan). Biosystems Diversity, 26(1), 56-61. doi: 10.15421/011809

The prime objective of the research was to list the important ethnomedicinal plants of Nawagai village, District Buner. During the survey, 44 plant species from 27 families were observed and collected from the targeted area of Khyber Pakhtunkhwa, Pakistan. Lamiaceae members were the most dominant (54\%) followed by members of Asteraceae (30\%), Poaceae (18\%) and Solanaceae (12\%). Relevant information such as field data, GPS coordinates family names, local names, therapeutic uses and plant habits were recorded for each species. For preservation purposes, specimens were mounted on herbarium sheets, and identified with the help of flora of Pakistan, flora of Australia and other relevant floristic records. During this research work all the collected specimens were preserved in the (BG\&H, UOM) Botanical Garden and Herbarium, the data were also provided to the Department of Botany, University of Malakand Dir (Lower), Khyber Pakhtunkhwa, Pakistan.
\end{abstract}

Keywords: medicinal plants; therapeutic uses; georeferenced data; District Bunir

\section{Introduction}

District Buner comes under the administrative umbrella of Malakand division. The word Buner is most probably taken from Sanskrit language which means "forest", since the area has some old growth coniferous forests patches present. The climate of the study area is of moderate nature. Buner covers an area of $1760 \mathrm{~km}^{2}$ lies between $34^{\circ} 09^{\prime}$ and $34^{\circ} 43^{\prime} \mathrm{N}$ latitudes and $72^{\circ} 10^{\prime}$ and $72^{\circ} 47^{\prime} \mathrm{E}$ longitudes. The village of Nawagai was chosen for the study as it provides easy access to the diverse representative vegetation of the district. Most of these plant species have medicinal value. Soil variation and diversification might be of the reason for this floristic diversity. The common types of soil found are sandy, clay and loamy textured.

Plants provide a wide range of products and services to people throughout the world. Most people of the third world countries rely on wild plants for their basic health care. The term "ethnobotany" was first introduced in 1896 by John Hershberger as the study of aromatic and wild plants used by primitive and aboriginal people. From that time, it has been defined as the traditional information of indigenous communities of the encompassing plant variety and the investigation of how the general population of a specific culture and area utilizes indigenous plants. Worldwide medicinal plants and medicines derived from them are used widely in traditional recipes and becoming popular in today's modern society. Ethnobotanical studies contribute to the knowledge of plant biodiversity, human awareness about the uses, applications, natural resources conservation and provide further social and scientific interventions for scientists (Parada et al., 2009). Ethnobotanical study helps the local community to establish the priorities for local use of plants for different aliments, this is also an effective source for conservation and cultural knowledge of the areas where these important plants occur (Ibrar et al., 2007).

Globally, over five thousand plant species belonging to the angiosperm group are used for medicinal purposes (Govaerts, 2001). Medicinal plants and their products have been used successfully for many diseases, both externally and internally. Medicinal plant material has persisted as the "treatment of choice" as it has no or few side effects
(Halberstein, 2005). Ethnobotany in Pakistan is not a highly profiled research area; however, many botanists have published research articles about medicinal plants. It has been reported that $84 \%$ of the population used traditional medicinal plants for many diseases (Shahzeb et al., 2013). Aromatic and wild medicinal plants are important tools for the treatment of different diseases (Bakoet al., 2005). Pakistan exhibit a rich history of traditional utilization of flora. Medicinal plants play a very important role in traditional health care of humans and animals. Extracts of medicinal plants are used in allopathic drugs (Hussain et al., 2010). In the national flora of Pakistan, more than $10 \%$ of medicinally important plant species were used in traditional treatments (Shinwari, 2010). Ethno-botanical information also helps ecologists, pharmacologists, taxonomists, watershed and wild life managers in their efforts for improving the economic status of the locals in remote areas (Ibrar et al., 2007).

In the study area, local peoples depend mostly upon medicinal plants, which provide a good source of primary health care material. The knowledge of use of particular medicinal plants for particular diseases is passed-on from generation after generation. Manan et al. (2007), reported an ethno-botanical review of Wari Sub-division Dir (U) with the main emphasis on the indigenous uses of the local plants. The inhabitants use medicinal plants on the advice of elders, such as wise men, herbalists, and traditional practitioners. They use them with the advice of nonqualified but professional traditional herbalists (Hakims), who have also gained some experience through apprenticeship with some registered practitioners. Medicinal plants are prescribed for for a wide range of diseases and ailments by qualified registered practitioners of the Unani system of medicine. These herbal medications were made by using water as a medium and administrated along with milk, ghee, oil, egg, sulphur, and butter etc. There are different steps to prepare herbal medicine, plant parts used in crushed form, in powders, in pastes, fresh, in decoctions, in juices, in extracts, as latex, in infusions, and as resin etc. (Abbasi et al., 2010). Medicinally important plants are divided into two broad types. Firstly, plants which are used by local physicians in different crude formulation to provide some relief to the local inhabitants in developing countries. Secondly, those plant which are in demand by pharmaceutical industries for 
extraction of their active ingredients (Hussain et al., 2008). Medicinal plants which are still widely used have been noted in Swat district. Some species seem to be adapted to wood-pasture, but vulnerable to overcollection, and in particular to deforestation. The better idea is to develop small-scale agroforestry systems to cultivate medicinally important plants for commercial and medicinal use by locals or with the help of the government (Akhtar et al., 2013).

\section{Materials and methods}

Field survey and collection of specimens was done in the study area of Nawagai. The data of use of medicinal plants was collected predominantly from old residents. Specimens of all the available species were collected along with their relevant field data and georeferenced location data (Fig. 1, Table 1-4).

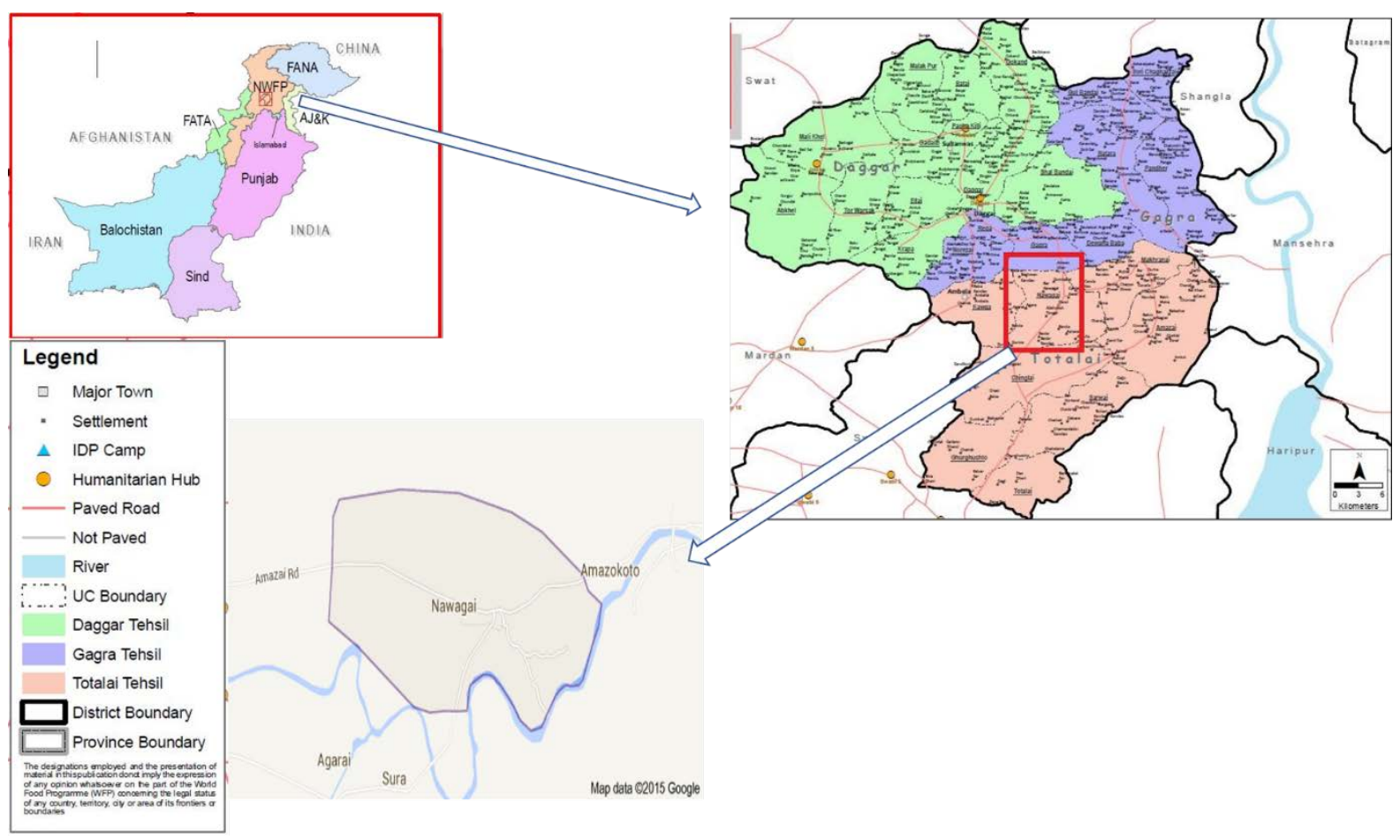

Fig. 1. Map of District Buner, Khyber Pakhtunkhwa

Table 1

List of GPS locations of selected plants

\begin{tabular}{ll}
\hline No & \multicolumn{1}{c}{ Species } \\
\hline 1 & Adiantum venustum D. Don \\
2 & Narcissus tazetta (L.) \\
3 & Hedera nepalensis K. Koch \\
4 & Calotropis procera (Aiton) W. T. Aiton \\
5 & Caralluma tubercullata N. E. Brown \\
6 & Taraxacum officinale Wigg. \\
7 & Xanthium strumarium (L.) \\
8 & Silybum marianum Gaertn. \\
9 & Carthamus oxycantha M. Bieb. \\
10 & Launea procumbense (Roxb.) Ramayya \& \\
& Rajjagopal
\end{tabular}

11 Conyza canadensis (L.) Corgn.

12 Berberis lycium Royle

13 Nasturtium officinale R. Br.

14 Cannabis sativa (L.)

15 Opuntia monacantha (Willd.) Haw.

15 f. variegata Hort

16 Euphorbia helioscopia (L.)

17 Trigonella foenum-graecum (L.)

18 Medicago sativa (L.)

19 Fumaria indica (Hausskn.) Pugsley

20 Ajuga bracteosa Wall. ex Benth

21 Mentha longifolia (L.)

22 Mentha spicata (L.)

23 Salvia moorcroftiana (L.)

24 Origanum vulgare (L.)

25 Isodon rugosus (Wall. ex Benth.) Codd.

26 Ocimum basilicum (L.)

27 Lathyrus aphaca (L.)

28 Otostegia limbata (Benth.) Boiss

29 Melia azedarach (L.)

30 Olea ferruginea Royle

\begin{tabular}{llll}
\hline No & Latitude & Longitude & Elevation \\
\hline 31 Oxalis corniculata (L.) & $34^{\circ} 24^{\prime} 38^{\prime \prime}$ & $72^{\circ} 33^{\prime} 12^{\prime \prime}$ & 686 \\
32 Piper nigrum (L.) & $34^{\circ} 24^{\prime} 35^{\prime \prime}$ & $72^{\circ} 33^{\prime} 23^{\prime \prime}$ & 682 \\
33 Plantago lanceolata (L.) & $34^{\circ} 24^{\prime} 02^{\prime \prime}$ & $72^{\circ} 33^{\prime} 29^{\prime \prime}$ & 665 \\
34 Polygonum barbatum (L.) & $34^{\circ} 25^{\prime} 24^{\prime \prime}$ & $72^{\circ} 34^{\prime} 14^{\prime \prime}$ & 815 \\
35 Rumex hastatus D. Don & $34^{\circ} 25^{\prime} 11^{\prime \prime}$ & $72^{\circ} 34^{\prime} 16^{\prime \prime}$ & 698 \\
36 Desmostachya bipinnata (L.) Stapf. & $34^{\circ} 24^{\prime} 08^{\prime \prime}$ & $72^{\circ} 33^{\prime} 41^{\prime \prime}$ & 672 \\
37 Punica granatum (L.) & $34^{\circ} 24^{\prime} 12^{\prime \prime}$ & $72^{\circ} 33^{\prime \prime} 28^{\prime \prime}$ & 680 \\
38 Rubus fruiticosus Agg. & $34^{\circ} 24^{\prime} 01^{\prime \prime}$ & $72^{\circ} 33^{\prime} 35^{\prime \prime}$ & 667 \\
39 Zanthxylum armatum DC. & $34^{\circ} 24^{\prime} 18^{\prime \prime}$ & $72^{\circ} 34^{\prime} 19^{\prime \prime}$ & 671 \\
40 Dodonaea viscose (L.) Jacq. & $34^{\circ} 24^{\prime} 56^{\prime \prime}$ & $72^{\circ} 24^{\prime} 54^{\prime \prime}$ & 807 \\
41 Verbascum thapsus (L.) & $34^{\circ} 23^{\prime} 54^{\prime \prime}$ & $72^{\circ} 33^{\prime} 23^{\prime \prime}$ & 675 \\
42 Solanum nigrum (L.) & $34^{\circ} 23^{\prime} 41^{\prime \prime}$ & $72^{\circ} 33^{\prime} 34^{\prime \prime}$ & 666 \\
43 Vitex negundo (L.) & $34^{\circ} 24^{\prime} 50^{\prime \prime}$ & $72^{\circ} 34^{\prime \prime} 26^{\prime \prime}$ & 674 \\
44 Verbena officinalis (L.) & $34^{\circ} 24^{\prime} 26^{\prime \prime}$ & $72^{\circ} 33^{\prime} 26^{\prime \prime}$ & 682 \\
\hline
\end{tabular}

Table 2

List of species with their family and local name and habit

\begin{tabular}{llll}
\hline \multicolumn{1}{c}{ Family } & \multicolumn{1}{c}{ Species } & \multicolumn{1}{c}{ Local name } & Habit \\
\hline Adiantaceae & Adiantum venustum D. Don & Sumbal & herb \\
Amaryllidaceae & Narcissus tazetta (L.) & Gule-e-Nargis herb \\
Araliaceae & Hedera nepalensis K. Koch & Ropai panra & shrub \\
Asclepiadaceae & Calotropis procera (Aiton) W. & Spalmai & shrub \\
Asclepiadaceae & Caralluma tubercullata N. E. Brown & Pamankay & herb \\
Asteraceae & Taraxacum officinale Wigg. & Ziar gulae & herb \\
Asteraceae & Xanthium strumarium (L.) & Gishkey & herb \\
Asteraceae & Silybum marianum Gaertn. & Bangi & shrub \\
Asteraceae & Carthamus oxycantha M. Bieb. & Kareeza & herb \\
Asteraceae & Launea procumbense (Roxb.) & Paiwaray & shrub \\
Asteraceae & Conyza canadensis (L.) Corgn. & Paleet & herb \\
Berberidaceae & Berberis lycium Royle & kwaray & shrub \\
Brassicaceae & Nasturtium officinale R. Br. & Talmeera & herb \\
Cannabaceae & Cannabis sativa (L.) & Bhang & shrub \\
Cactaceae & Opuntia monacantha (Willd.) Haw. & Zooqam & shrub \\
Euphorbiaceae & Euphorbia helioscopia (L.) & Peryan dholay herb \\
\hline
\end{tabular}




\begin{tabular}{|c|c|c|c|}
\hline Family & Species & Local name & Habit \\
\hline Fabaceae & Trigonella foenum-graecum (L.) & Malkhoza & herb \\
\hline Fabaceae & Medicago sativa (L.) & Shpeshty & herb \\
\hline Fumariaceae & Fumaria indica (Hausskn.) Pugsley & Papra & herb \\
\hline Lamiaceae & Ajuga bracteosa Wall. ex Benth & Booti & herb \\
\hline Lamiaceae & Mentha longifolia (L.) & Villanay & herb \\
\hline Lamiaceae & Mentha spicata (L.) & Podeena & herb \\
\hline Lamiaceae & Salvia moorcroftiana (L.) & Kharghwag & herb \\
\hline Lamiaceae & Origanum vulgare (L.) & Shamakay & shrub \\
\hline Lamiaceae & Isodon rugosus (Wall. ex Benth.) Codd. & Sperkay & shrub \\
\hline Lamiaceae & Ocimum basilicum (L.) & Kashmali & shrub \\
\hline Lamiaceae & Lathyrus aphaca (L.) & Kurkamany & herb \\
\hline Lamiaceae & Otostegia limbata (Benth.) Boiss & Spen Azghay & herb \\
\hline Meliaceae & Melia azedarach (L.) & Tora bakyanra & tree \\
\hline Oleaceae & Olea ferruginea Royle & Khona & tree \\
\hline Oxalidaceae & Oxalis corniculata (L.) & Tarookay & herb \\
\hline Piperaceae & Piper nigrum (L.) & Mirch & tree \\
\hline Plantaginaceae & Plantago lanceolata (L.) & Jabai & herb \\
\hline Polygonaceae & Polygonum barbatum (L.) & Palpolak & herb \\
\hline Polygonaceae & Rumex hastatus D. Don & Tarookay & herb \\
\hline Poaceae & Desmostachya bipinnata (L.) Stapf. & Drub & herb \\
\hline Punicaceae & Punica granatum (L.) & Ananghori & tree \\
\hline Rosaceae & Rubus fruiticosus Agg. & Karwara & shrub \\
\hline Rutaceae & Zanthxylum armatum DC. & Dambara & tree \\
\hline Sapindaceae & Dodonaea viscose (L.) & Ghwarasky & shrub \\
\hline Scrophulariaceae & Verbascum thapsus (L.) & Khardag & herb \\
\hline Solanaceae & Solanum nigrum (L.) & Kachmachu & herb \\
\hline Verbenaceae & Vitex negundo (L.) & Marvandai & shrub \\
\hline Verbenaceae & Verbena officinalis (L.) & Shamakay & shrub \\
\hline
\end{tabular}

Table 3

List of plants name and their abbreviations

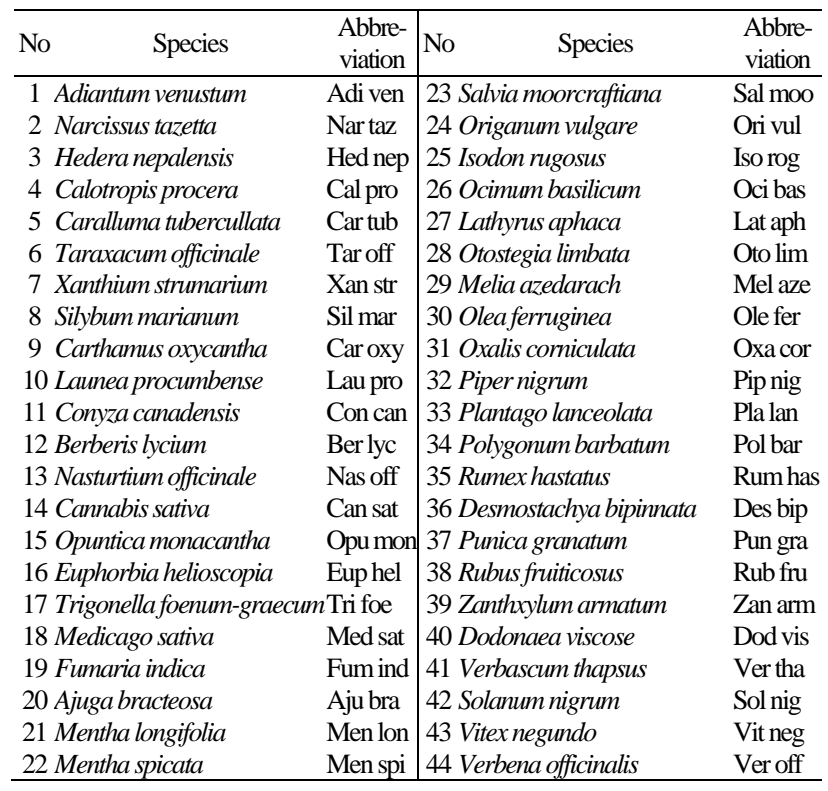

After collection, the plants were pressed to obtain good herbarium specimens. They were pressed before their wilting in newspaper sheets. The newspapers were changed every 24 hours. After pressing and drying, the specimens were mounted on herbarium sheets for obtaining morphological data for their identification to species level. After their identification, all the available information about the taxa were transferred upon their respective herbarium sheet. The plant species were either identified directly in the field or after processing at the Botanical Garden and Herbarium, University of Malakand Chakdara, Dir lower, Khyber Pakhtunkhwa with the help of flora of Pakistan, flora of Australia and other relevant published sources. The voucher specimen of each species was deposited in the Herbarium and Department of Botany, University of Malakand, Pakistan.

\section{Results}

The ethnomedicinal flora of Nawagai Village consists of 44 species belonging to 27 families. Among the collected plants, 9 plants belong to the family Lamiaceae, 6 to the family Asteraceae, 2 to Asclepiadaceae, Polygonaceae, Fabaceae and Verbenaceae. One species was recorded for Adiantaceae, Amaryllidaceae, Araliaceae, Berberidaceae, Brassicaceae, Cactaceae, Cannabaceae, Euphorbiaceae, Meliaceae, Oleaceae, Oxalidaceae, Piperaceae, Plantaginaceae, Poaceae, Punicaceae, Rosaceae, Rutaceae, Sapindaceae, Scrophulariaceae and Solanaceae (Fig. 2).

Table 4

List of therapeutic uses abbreviation

\begin{tabular}{|c|c|c|c|c|c|}
\hline No & Full name & $\begin{array}{l}\text { Abbre- } \\
\text { viation }\end{array}$ & No & Full name & $\begin{array}{l}\text { Abbre- } \\
\text { viation }\end{array}$ \\
\hline 1 & stomach-ache & sto & 38 & tumours & tum \\
\hline 2 & vermifuge & ver & 39 & anaemia & ane \\
\hline 3 & diurietries & diu & 40 & digestive & dig \\
\hline 4 & astringent & ast & 41 & appetite & app \\
\hline 5 & diarrhrea & dia & 42 & lung infections & lun \\
\hline 6 & expectorant & $\exp$ & 43 & cancer & can \\
\hline 7 & cough & $\mathrm{COU}$ & 44 & heartburn & hea \\
\hline 8 & tonic & ton & 45 & sore throat & thr \\
\hline 9 & stimulant & sti & 46 & antioxidant & ant \\
\hline 10 & purgative & pur & 47 & antimicrobial & ant \\
\hline 11 & rheumatism & rhe & 48 & cns disorders & cns \\
\hline 12 & toothache & too & 49 & ant dyspeptic & ant \\
\hline 13 & honey formation & hon & 50 & cholagogue & cho \\
\hline 14 & fever & fev & 51 & diaphoretic & dia \\
\hline 15 & blood purifying & blo & 52 & abdominal cramps & abd \\
\hline 16 & dysentery & dys & 53 & achene’s & ach \\
\hline 17 & laxative & $\operatorname{lax}$ & 54 & antiseptic & ant \\
\hline 18 & carminative & car & 55 & analgesic & ana \\
\hline 19 & sedative & sed & 56 & aromatic & aro \\
\hline 20 & asthma & ast & 57 & ear pain & ear \\
\hline 21 & emetic & eme & 58 & burns & bur \\
\hline 22 & diabetes & dia & 59 & gum diseases & gum \\
\hline 23 & pulmonary & pul & 60 & hysteria & hys \\
\hline 24 & skin disease & ski & 61 & antiperiodic & ant \\
\hline 25 & hypertension & hyp & 62 & curing of flu & cur \\
\hline 26 & kidney & kid & 63 & muscle relaxation & mus \\
\hline 27 & liver disease & liv & 64 & mouth diseases & mou \\
\hline 28 & flow & flo & 65 & fish poison & fis \\
\hline 29 & haemorrhage & hae & 66 & strangury & str \\
\hline 30 & apparent & app & 67 & vaginal discharges & vag \\
\hline 31 & sudorific & sud & 68 & vesicle calculi & ves \\
\hline 32 & cnolagugue & cno & 69 & biliousness & bil \\
\hline 33 & micturition & mic & 70 & bladder diseases & bla \\
\hline 34 & wound & wou & 71 & swelling & swe \\
\hline 35 & anti-scorbic & ant & 72 & opthalmopathy & opt \\
\hline 36 & narcotic & nor & 73 & odontalgia & odo \\
\hline 37 & urinary & uri & 74 & verminosis & ver \\
\hline
\end{tabular}

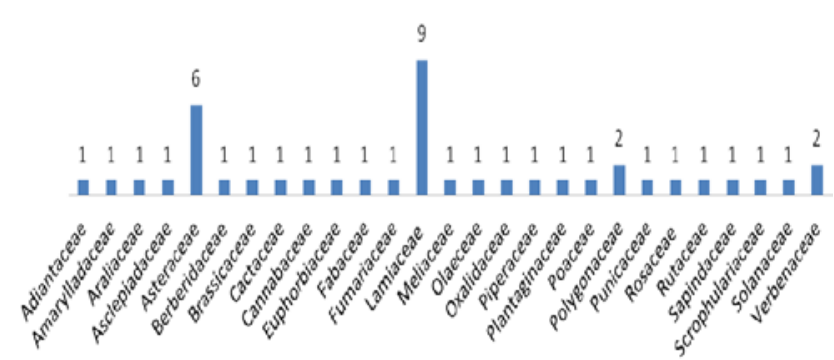

Fig. 2. Frequency distribution of selected plant families

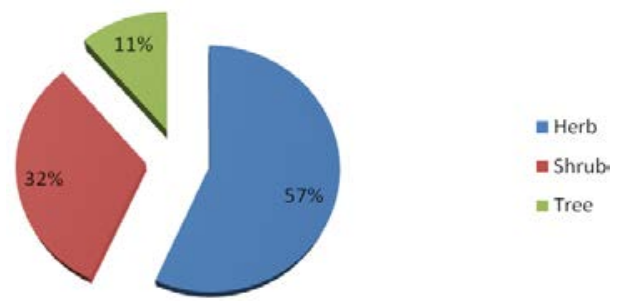

Fig. 3. Frequency distribution for habits of selected plant species

Based on their status, these plants were further divided into herbs, shrubs and trees (Fig. 3). 
Table 5

List of plant uses against different aliments

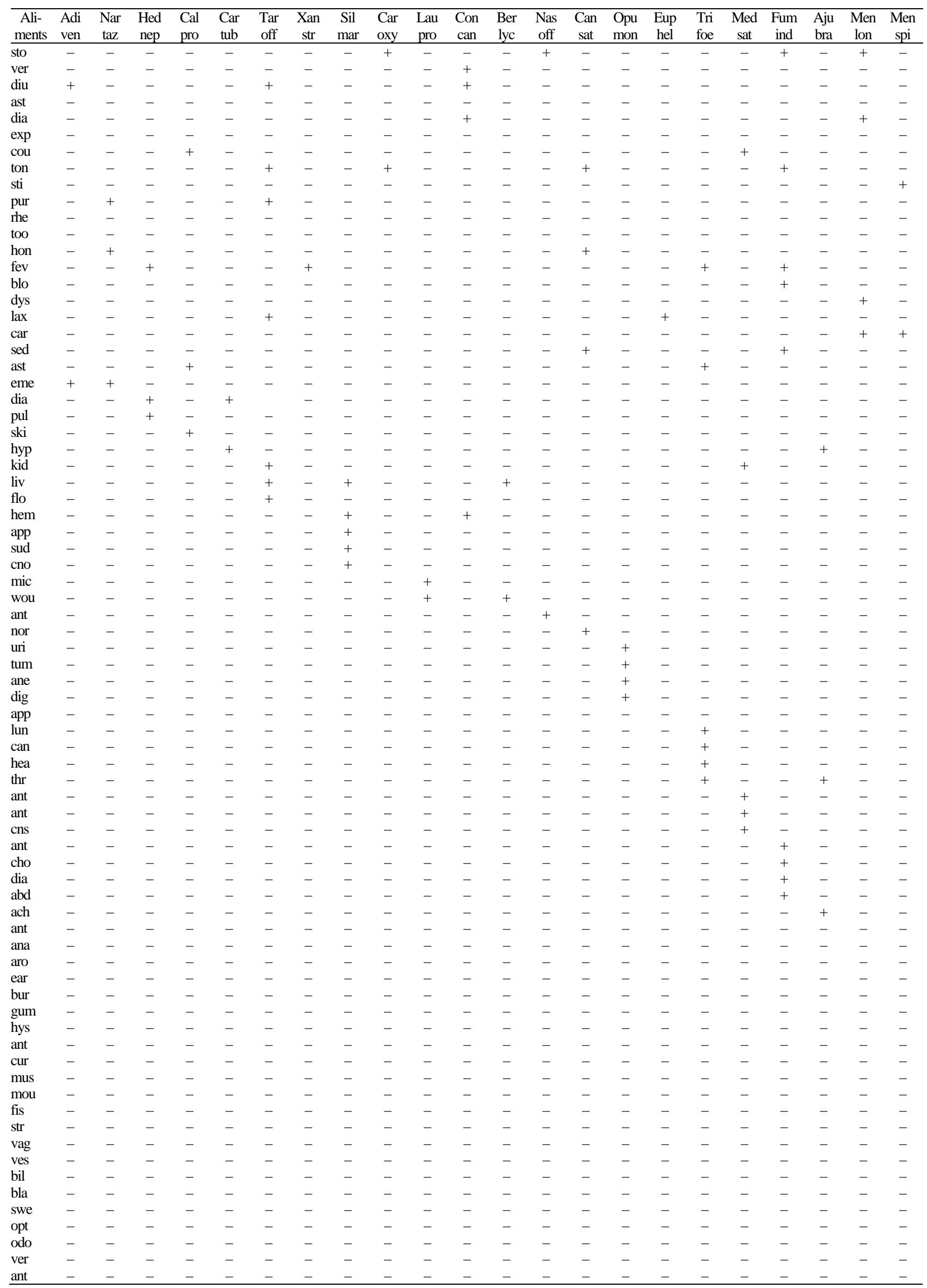


Table 6

List of plant uses against different aliments

\begin{tabular}{|c|c|c|c|c|c|c|c|c|c|c|c|c|c|c|c|c|c|c|c|c|c|c|}
\hline $\begin{array}{c}\text { Ali- } \\
\text { ments }\end{array}$ & $\begin{array}{c}\text { Sal } \\
\text { moo }\end{array}$ & $\begin{array}{l}\text { Ori } \\
\text { vul }\end{array}$ & $\begin{array}{l}\text { Iso } \\
\text { rog }\end{array}$ & $\begin{array}{l}\text { Oci } \\
\text { bas }\end{array}$ & $\begin{array}{l}\text { Lat } \\
\text { aph }\end{array}$ & $\begin{array}{l}\text { Oto } \\
\text { lam }\end{array}$ & $\begin{array}{l}\text { Mel } \\
\text { aze }\end{array}$ & $\begin{array}{l}\text { Ole } \\
\text { fer }\end{array}$ & $\begin{array}{l}\text { Oxa } \\
\text { cor }\end{array}$ & $\begin{array}{l}\text { Pip } \\
\text { nig }\end{array}$ & $\begin{array}{l}\text { Pla } \\
\text { lan }\end{array}$ & $\begin{array}{l}\text { Pol } \\
\text { bar }\end{array}$ & $\begin{array}{c}\text { Rum } \\
\text { has }\end{array}$ & $\begin{array}{l}\text { Des } \\
\text { bip }\end{array}$ & $\begin{array}{c}\text { Pun } \\
\text { gra }\end{array}$ & $\begin{array}{l}\text { Rub } \\
\text { fru }\end{array}$ & $\begin{array}{l}\text { Zan } \\
\text { arm }\end{array}$ & $\begin{array}{c}\text { Dod } \\
\text { vis }\end{array}$ & $\begin{array}{l}\begin{array}{l}\text { Ver } \\
\text { tha }\end{array} \\
\end{array}$ & $\begin{array}{l}\text { Sol } \\
\text { nig }\end{array}$ & $\begin{array}{l}\text { Vit } \\
\text { neg }\end{array}$ & $\begin{array}{l}\text { Ver } \\
\text { off }\end{array}$ \\
\hline sto & - & - & - & - & - & - & - & - & + & - & - & - & + & - & - & - & - & - & - & - & - & - \\
\hline ver & - & - & - & - & - & - & - & - & + & - & - & - & - & - & - & - & - & - & - & - & - & - \\
\hline diu & - & + & - & - & - & - & - & + & - & - & - & - & + & - & - & + & - & - & - & + & - & - \\
\hline ast & - & - & - & - & - & - & - & - & - & - & - & - & - & - & - & - & - & + & - & - & - & - \\
\hline dia & + & - & - & - & - & - & - & - & - & - & - & - & - & - & - & + & - & - & + & _ & _ & + \\
\hline $\exp$ & - & - & - & - & - & - & - & - & - & - & - & - & - & - & - & - & - & - & - & + & - & - \\
\hline cou & + & - & - & - & - & - & - & - & - & - & - & - & - & - & + & + & - & - & + & - & - & - \\
\hline ton & - & - & - & - & - & - & - & - & - & - & - & - & - & - & - & - & + & - & - & + & - & - \\
\hline sti & - & - & - & - & - & - & - & - & - & - & - & - & - & - & - & - & - & - & - & - & - & - \\
\hline pur & - & - & - & - & - & - & - & - & - & - & - & - & - & - & - & - & - & - & - & - & - & - \\
\hline rhe & - & - & - & - & - & - & - & - & - & - & - & - & - & - & - & - & - & + & - & - & - & - \\
\hline too & - & + & - & - & - & - & - & + & + & - & - & - & - & - & - & - & + & - & - & - & - & - \\
\hline hon & - & - & - & - & - & - & + & - & - & - & - & - & - & - & - & - & - & - & - & - & - & - \\
\hline fev & - & - & - & - & - & - & - & - & + & - & - & - & - & - & - & + & + & - & - & - & - & + \\
\hline blo & - & - & - & - & - & - & + & - & - & - & - & - & + & - & + & - & - & - & - & - & - & + \\
\hline dys & + & - & - & + & - & - & - & - & + & - & + & - & - & - & + & - & - & - & + & - & - & - \\
\hline lax & - & - & - & - & - & - & - & - & - & - & + & - & - & - & + & - & - & - & - & - & - & - \\
\hline $\mathrm{car}$ & - & - & - & + & - & - & - & - & - & - & - & - & + & - & - & + & - & - & - & + & - & - \\
\hline sed & - & - & - & - & - & - & - & - & - & - & - & - & - & + & - & - & - & - & - & - & - & - \\
\hline ast & - & - & - & - & - & - & - & - & - & - & - & - & - & + & - & - & - & - & - & - & - & - \\
\hline eme & - & - & - & - & - & - & + & - & - & - & - & - & - & - & - & - & - & - & - & - & - & - \\
\hline dia & - & - & - & - & - & - & + & + & - & - & - & - & - & + & - & - & - & - & - & - & - & - \\
\hline pul & + & - & - & - & - & - & - & - & - & - & - & - & - & - & - & - & - & - & + & - & - & - \\
\hline ski & - & - & - & - & - & - & - & - & - & - & - & - & - & + & + & - & - & - & - & + & - & - \\
\hline hyp & - & - & - & - & - & - & - & - & - & - & - & - & - & - & - & - & - & - & - & - & - & - \\
\hline kid & - & - & - & - & - & - & - & - & - & - & - & - & - & - & - & - & - & - & - & - & - & - \\
\hline liv & - & - & - & - & - & - & - & - & - & - & - & - & - & - & - & - & - & - & - & - & - & - \\
\hline flo & - & - & - & - & - & - & - & - & - & - & - & - & - & - & - & - & - & - & - & - & - & - \\
\hline hem & - & - & - & - & - & - & - & - & - & - & - & - & - & - & - & - & - & - & - & - & - & - \\
\hline арр & - & - & - & - & - & - & - & - & - & - & - & - & - & - & - & - & - & - & - & - & - & - \\
\hline sud & - & - & - & - & - & - & - & - & - & - & - & - & - & - & - & - & - & - & - & - & - & - \\
\hline cno & - & - & - & - & - & - & - & - & - & - & - & - & - & - & - & - & - & - & - & - & - & - \\
\hline mic & - & - & - & - & - & - & - & - & - & - & - & - & - & - & - & - & - & - & - & - & - & - \\
\hline wou & + & - & - & - & - & + & - & - & - & - & + & - & - & - & - & - & - & - & + & + & - & - \\
\hline ant & - & - & - & - & - & - & - & - & - & - & - & - & - & - & - & - & - & - & - & - & - & - \\
\hline nor & - & - & - & - & - & - & - & - & - & - & - & - & - & - & - & - & - & - & - & - & - & - \\
\hline uri & - & - & - & - & - & - & - & - & - & - & - & - & - & - & - & - & - & - & - & - & - & - \\
\hline tum & - & - & - & - & - & - & - & - & - & - & - & - & - & - & - & - & - & - & - & - & - & - \\
\hline ane & - & - & - & - & - & - & - & - & - & - & - & - & - & - & - & - & - & - & - & - & - & - \\
\hline dig & - & - & - & - & - & - & - & - & - & - & - & - & - & - & - & - & - & - & - & - & - & - \\
\hline app & - & - & - & - & - & - & - & - & - & - & - & - & - & - & - & - & - & - & - & - & - & - \\
\hline lun & - & - & - & - & - & - & - & - & - & - & - & - & - & - & - & - & - & - & - & - & - & - \\
\hline can & - & - & - & - & - & - & - & - & - & - & - & - & - & - & - & - & - & - & - & - & - & - \\
\hline hea & - & - & - & - & - & - & - & - & - & - & - & - & - & - & - & - & - & - & - & - & - & - \\
\hline thr & - & - & - & - & - & - & - & + & - & - & - & - & - & - & - & - & - & - & - & - & - & - \\
\hline ant & - & - & - & - & - & - & - & - & - & - & - & - & - & - & - & - & - & - & - & - & - & - \\
\hline ant & - & - & - & - & - & - & - & - & - & - & - & - & - & - & - & - & - & - & - & - & - & - \\
\hline cns & - & - & - & - & - & - & - & - & - & - & - & - & - & - & - & - & - & - & - & - & - & - \\
\hline ant & - & - & - & - & - & - & - & - & - & - & - & - & - & - & - & - & - & - & - & - & - & - \\
\hline cho & - & - & - & - & - & - & - & - & - & - & - & - & - & - & - & - & - & - & - & - & - & - \\
\hline dia & - & - & - & - & - & - & - & - & - & - & - & - & - & - & - & - & - & - & - & - & - & - \\
\hline abd & - & - & - & - & - & - & - & - & - & - & - & - & + & - & - & - & - & - & - & - & - & - \\
\hline ach & - & - & - & - & - & - & - & - & - & - & - & - & - & - & - & - & - & - & - & - & - & - \\
\hline ant & + & - & + & - & - & - & - & + & - & - & - & - & - & - & - & - & - & - & + & - & - & - \\
\hline ana & + & - & - & - & - & - & - & - & - & - & - & - & - & - & - & - & - & - & + & - & - & - \\
\hline aro & - & - & + & - & - & - & - & - & - & - & - & - & - & - & - & - & + & - & - & - & - & - \\
\hline ear & - & - & - & + & - & - & - & - & - & - & - & - & - & - & - & - & - & - & - & - & - & - \\
\hline bur & - & - & - & - & + & - & - & - & - & - & - & - & - & - & - & - & - & + & - & - & - & - \\
\hline gum & - & - & - & - & - & + & - & - & - & - & - & - & - & - & - & - & + & - & - & - & - & - \\
\hline hys & - & - & - & - & - & - & + & - & - & - & - & - & - & - & - & - & - & - & - & - & - & - \\
\hline ant & - & - & - & - & - & - & - & + & - & - & - & - & - & - & - & - & - & - & - & - & - & - \\
\hline cur & - & - & - & - & - & - & - & - & - & + & - & - & - & - & - & - & - & - & - & - & - & - \\
\hline mus & - & - & - & - & - & - & - & - & - & + & - & - & - & - & - & - & - & - & - & - & - & - \\
\hline mou & - & - & - & - & - & - & - & - & - & - & + & - & - & - & - & - & - & - & - & - & - & - \\
\hline fis & - & - & - & - & - & - & - & - & - & - & - & + & - & - & - & - & - & - & + & - & - & - \\
\hline str & - & - & - & - & - & - & - & - & - & - & - & - & - & + & - & - & - & - & - & - & - & - \\
\hline vag & - & - & - & - & - & - & - & - & - & - & - & - & - & + & - & - & - & - & - & - & - & - \\
\hline ves & - & - & - & - & - & - & - & - & - & - & - & - & - & + & - & - & - & - & - & - & - & - \\
\hline bil & - & - & - & - & - & - & - & - & - & - & - & - & - & + & - & - & - & - & - & - & - & - \\
\hline bla & - & - & - & - & - & - & - & - & - & - & - & - & - & + & - & - & - & - & - & - & - & - \\
\hline swe & - & - & - & - & - & - & - & - & - & - & - & - & - & - & - & - & - & + & - & - & - & - \\
\hline opt & - & - & - & - & - & - & - & - & - & - & - & - & - & - & - & - & - & - & - & - & + & - \\
\hline odo & - & - & - & - & - & - & - & - & - & - & - & - & - & - & - & - & - & - & - & - & + & - \\
\hline ver & - & - & - & - & - & - & - & - & - & - & - & - & - & - & - & - & - & - & - & - & + & - \\
\hline ant & - & - & - & - & - & - & - & - & - & - & - & - & - & - & - & - & - & - & - & - & + & _- \\
\hline
\end{tabular}


As per medicinal use of the plants, 74 therapeutic classes as shown in Table 5, 6 were formed, in which 8 plants are used for fever, 7 as diuretics, 7 as cure for dysentery and 7 helping in wound healing, 6 as cough suppressant, 6 used as tonic, 6 plants are also used for curing diarrhea, 6 plants are used for the treatment of stomach ache and 6 species are used for curing diabetes, 5 as blood purifying, 4 as antiseptic, 4 used as laxative, 4 used for skin disease and 4 plants are used in toothache, 3 as honey bee plants, 3 as sedatives, 3 used in asthmatic situation, 3 as cure for pulmonary and liver disease, 2 plants are used as vermifuge, 2 as astringent, 2 as purgative, also 2 plants are used as antiemetic, 2 as hypertension remedy, also 2 species used in kidney problems, 2 as haemorrhage, 2 used in abdominal cramps, 2 as analgesic, 2 as aromatic, 2 used for burns, 2 as gum disease, and 2 plants used as fish poison. And only one plant species is used for each of the following: stimulant, rheumatism, flow, apparent, sudorific, micturition, antiscorbic, narcotic, urinary, tumors, anemia, digestive, appetite, lung infections, cancer, heartburn, sore throat sore, antioxidant, antimicrobial, CNS disorders, ant dyspeptic, cholagogue, diaphoretic, achene's, ear pain, hysteria, antiperiodic, curing of flu, muscle relaxation, mouth diseases, strangury, vaginal discharges, vesicle calculi, biliousness, bladder diseases, swelling, ophthalmopathy, odontalgia, verminosis and anti-inflammatory.

\section{Discussion}

The study indicates that the local people use various native plants for different purposes such as for fuel e.g. Berberis lycium, Cannabis sativum and as fodder for animals like Plantago lanceolata, Oxalis corniculata, Medicago sativa. Some people used these plants as medicine against different diseases, Ajuga bracteosa is used for abdominal pain, Verbascum thapsus for external wound healing and Berberis lycium for gum diseases. Extraction of leaves of Hedera nepalensis locally used to maintain level of diabetes. The leaves of Mentha longifolia, Isodon rogusus, Ajuga bracteosa, Salvia moorcroftiana were used for the treatment of abdominal pain, digestive disorders, woundshealing. In the Indian system of medicines Zanthoxylum armatum is used as carminative, stomachic and treatment of toothache (Prasanta \& Mukherjee, 2011).

Medicinal plants have no side effects so use of medicinal plants or their resources should be rapidly increased, and they should be easily available and this is only source of health care available to the middleclass population (Acharya et al., 2009). Ahmad et al. (2014) reported a total of 50 plant species belonging to 48 genera of 35 families from Chail valley Swat. Umair et al. (2017) listed 85 species belonging to 71 genera, and 34 families were documented along with their ethnomedicinal uses. A total of 163 plant species belonging to 73 families were reported with the help of standardized questionnaires for their traditional, medicinal and economic uses (Sher et al., 2014). A total of 200 local inhabitants were found utilizing 52 plants of 37 families for various ethnobotanical purposes, notably as timber wood, fodder and forage for cattle, condiments, for shade, usage in construction and agricultural tool making etc. (Hassan et al., 2015). Khan and Musharaf (2015) also reported 34 plant species belonging to 20 families from tehsil Thakht bhai, District: Mardan. An ethnobotanical survey was conducted in Tirat Valley, Swat District, Pakistan, reporting 65 species belonging to 35 families, from the Valley which belonged to the ethnomedical domain of the area (Ali et al., 2017). The author found that Myrtus communis is a bushy, aromatic, ever-green shrub, belonging to the family Myrtaceae, is commonly found in Bajaur Agency (FATA) Pakistan.

Our study indicated that local people of the area used different parts of medicinal plants. Taraxacum officinale is used in many traditional medical systems particularly in Asia, Europe, and North America, the root being primarily considered a gastrointestinal remedy supporting digestion and liver function, while the leaf is used as a diuretic and bitter digestive stimulant (Yarnell \& Abascal, 2009).

\section{References}

Abbasi, A. M., Khan, M. A., Ahmad, M., Zafar, M., Jahan, S., \& Sultana, S. (2010). Ethnopharmacological application of medicinal plants to cure skin diseases and in folk cosmetics among the tribal communities of North-West Frontier Province, Pakistan. Journal of Ethnopharmacology, 128(2), 322-335.

Acharya, K., Chaudhary, R., \& Vetaas, O. (2009). Medicinal plants of Nepal: Distribution pattern along an elevational gradient and effectiveness of existing protected areas for their conservation. Banko Janakari, 19(1), 16-21.

Ahmad, M., Sultana, S., Fazl-i-Hadi, S., ben Hadda, T., Rashid, S., Zafar, M., Khan, M., Khan, M. P., \& Yaseen, G. (2014). An ethnobotanical study of medicinal plants in high mountainous region of Chail valley (District SwatPakistan). Journal of Ethnobiology and Ethnomedicine, 10(1), 36.

Akhtar, N., Rashid, A., Murad, W., \& Bergmeier, E. (2013). Diversity and use of ethno-medicinal plants in the region of Swat, North Pakistan. Journal of Ethnobiology and Ethnomedicine, 9(1), 25.

Ali, K., Khan, N., Rahman, I. U., Ahmad, S., Khan, W., Ali, M., \& Sheday, A. A. (2017). Climate change: An evil or an opportunity-evidence from the Hindu Kush Mountain system of Pakistan. International Journal of Biosciences, 11(2), 99-105.

Ali, K., Khan, S., Khan, N., Khan, W., Rahman, I. U., Ullah, F., Ali, K., \& Nisar, M. (2017). Ethnobotanical and ecological study of Punica granatum in Dir district, Khyber Pakhtunkhwa, Pakistan. Regulatory Mechanisms in Biosystems, 8(4), 656-661.

Ali, K., Ullah, F., Khan, N., Rahman, I. U., Ullah, S., Khan, W., Ali, M., Uddin, N., \& Nisar, M. (2017). Ethnobotanical and ecological study of Myrtus communis (L.) in Bajaur agency (FATA) Khyber-Pakhtunkhwa, Pakistan. Journal of Biodiversity and Environmental Sciences, 11(1), 152-164.

Ali, M., Begum, R., Ali, K., Nisar, M., Khan, W., Hassan, N., Uddin, N., Wadood, S. F., Zaman, S. U., Rahman, I. U., \& Khan, N. (2017). Ethnobotanical survey of wild medicinal plants of Tirat Valley district Swat, Khyber-Pakhtunkhwa. Journal of Biodiversity and Environmental Sciences, 11(3), 91-101.

Bako, S. P., Bakfur, M. J., John, I., \& Bala, E. (2005). Ethnomedicinal and phytochemical profile of some savanna plant species in Nigeria. International Joumal of Botany, 1(2), 147-150.

Govaerts, R., \& Govaerts, R. (2001). How many species of seed plants are there? Taxon, 50(4), 1085

Habib-Ul-Hassan, W. M., Ahmad, N., Tariq, A., Khan, I., Akhtar, N., \& Jan, S. (2015). Indigenous uses of the plants of Malakand valley, district Dir (Lower), Khyber Pakhtunkhwa, Pakistan. Pakistan Journal of Weed Sciences Resources, 21(1), 83-99.

Halberstein, R. A. (2005). Medicinal plants: Historical and cross-cultural usage patterns. Annals of Epidemiology, 15(9), 686-699.

Hussain, S. Z., Malik, R. N., Javaid, M., \& Bibi, S. (2008). Ethnobotanical properties and uses of medicinal plants of Morgah Biodiversity Park, Rawalpindi. Pakistan Journal of Botany, 40(5), 1897-1911.

Ibrar, M., Hussain, F., \& Sultan, A. (2007). Ethnobotanical studies on plant resources of Ranyal Hills, District Shangla, Pakistan. Pakistan Journal of Botany, 39(2), 329-337.

Ikram, S., Bhatti, K. H., \& Parvaiz, M. (2014). Ethnobotanical studies of aquatic plants of district Sialkot Punjab (Pakistan). Journal of Medicinal Plants Studies, 2(1), 58-63.

Khan, M., \& Musharaf, S. (2015). Ethnomedicinal and conservation status of plant species in Tehsil Takht Bhai, District Mardan, Pakistan. International Letters of Natural Sciences, 37, 18-29.

Manan, Z., Sirajuddin, A., Razzaq, M., \& Islam, M. (2007). Diversity of medicinal plants in Wari subdivision District upper Dir, Pakistan. Pakistan Journal of Plant Sciences, 13, 21-28.

Parada, M., Carrió, E., Bonet, M. À., \& Vallès, J. (2009). Ethnobotany of the Alt Empordà region (Catalonia, Iberian Peninsula). Journal of Ethnopharmacology, 124(3), 609-618.

Shahzeb, S., Mehmood, R., khan, U., \& Khan, S. U. (2013). Use of medicinal plants of District Bannu in Unani Medicines. International Journal of Herbal Medicine, 1(2), 81-88.

Shinwari, Z. K. (2010). Medicinal plants research in Pakistan. Joumal of Medicinal Plants Resources, 4(3), 161-176.

Umair, M., Altaf, M., \& Abbasi, A. M. (2017). An ethnobotanical survey of indigenous medicinal plants in Hafizabad district, Punjab-Pakistan. PLoS One, 12(6), $\mathrm{e} 0177912$.

Zaman, S., Farrukh, H., \& Muhammad, I. (2014). Traditional knowledge on plant resources of Ashezai and Salarzai Valleys, District Buner, Pakistan. African Journal of Plant Science, 8(1), 42-53. 